COMMUN. IN PARTIAL DIFFERENTIAL EQUATIONS, 27(5\&6), 1103-1137 (2002)

\title{
WELL-POSEDNESS AND GLOBAL ATTRACTORS FOR LIQUID CRYSTALS ON RIEMANNIAN MANIFOLDS
}

\author{
Steve Shkoller* \\ Department of Mathematics, University of California, \\ Davis, CA 95616
}

\begin{abstract}
We study the coupled Navier-Stokes Ginzburg-Landau model of nematic liquid crystals introduced by F.H. Lin, which is a simplified version ofthe Ericksen-Leslie system. We generalize the model to compact $n$-dimensional Riemannian manifolds, deriving the system from a variational principle, and provide a very simple proof of local well-posedness for this coupled system using a contraction mapping argument. We then prove that this system is globally well-posed and has compact global attractors when the dimension of the manifold $M$ is two. A small data result in $n$ dimensions follows easily. Finally, we introduce the Lagrangian averaged liquid crystal equations, which arise from averaging the Navier-Stokes fluid motion over small spatial scales in the variational principle. We show that this averaged system is globally well-posed and has compact global attractors even when $M$ is threedimensional.
\end{abstract}

*E-mail: shkoller@math.ucdavis.edu 
Key Words: Navier-Stokes; Ginzburg-Landau; Liquid crystals

1991 Mathematics Subject Classification: 35Q30; 35Q72; $58 \mathrm{~J} 35$

\section{INTRODUCTION}

Nematic liquid crystals are well-studied and interesting examples of anisotropic non-Newtonian fluids. A liquid crystal is a phase of a material between the solid and liquid phases. The solid phase has strong intermolecular forces that keep the molecular position and orientation fixed, while in the liquid phase, the molecules neither occupy a specific average position nor do they remain in any particular orientation; the nematic liquid crystal phase does not have any positional order, but does possess a certain amount of orientational order. This phase is described by a velocity field, as well as a director field that describes locally the averaged direction or orientation of the constituent molecules. In this paper, we shall analyze the behavior of a certain model of nematic liquid crystals on compact Riemannian manifolds.

We let $(M, g)$ denote a smooth, compact, connected, $n$-dimensional Riemannian manifold with smooth (possibly empty) boundary $\partial M$. If $\partial M=\emptyset$, then we assume that the Euler characteristic $\chi(M)$ does not vanish. We study the following system of nonlinear partial differential equations:

$$
\begin{aligned}
& u_{t}+\nabla_{u} u=-\operatorname{grad} p+v \operatorname{Div} \operatorname{Def} u-\lambda \operatorname{Div}\left(\nabla d^{T} \cdot \nabla d\right), \\
& \operatorname{div} u(t, x)=0, \\
& d_{t}+\nabla_{u} d=\gamma\left(\hat{\Delta} d-\frac{1}{\varepsilon^{2}}\left(|d|^{2}-1\right) d\right), \\
& u=0 \quad \text { on } \partial \mathrm{M}, d=h \text { on } \partial M \quad g(h, h)=1 \text { or } \partial M=\emptyset, \\
& u(0, x)=u_{0}, \quad d(0, x)=d_{0} \quad \text { and } d_{0 \mid \partial M}=h \quad \text { if } \partial M \neq \emptyset .
\end{aligned}
$$

Here $u(t, x)$ and $d(t, x)$ are time-dependent vector fields on $M, \nabla$ denotes the Levi-Civita covariant derivative associated to the Riemannian metric $g$, Def $u=(1 / 2)\left(\nabla u+\nabla u^{T}\right)$ denotes the (rate of) deformation tensor, the superscript $(\cdot)^{T}$ denotes the transpose, $\hat{\Delta}$ denotes the rough Laplacian of $g$ defined in (2.4), and $\nu, \lambda, \gamma$ are positive constants. In the case that $M$ is flat, an open subset of Euclidean space for instance, then $\nabla$ is the componentwise gradient, and $\hat{\Delta}$ is the componentwise Laplacian given in coordinates $x^{i}$ by $\hat{\Delta}=\sum_{i=1}^{n}\left(\partial^{2} / \partial x^{i} \partial x^{i}\right)$. The system of Eq. (1.1) is the simplified EricksenLeslie model (Refs. $[9,10,13]$ ) of nematic liquid crystals first introduced by 
F.H. Lin in Ref. [14] and later analyzed by F.H. Lin and C. Liu in Refs. $[16,17]$. Beautiful numerical simulations can be found in Ref. [21].

This system couples the Navier-Stokes (NS) equations with the Ginzburg-Landau (GL) penalization of the harmonic map heat flow. The vector field $u(t, x)$ is the velocity field of the fluid, while $d(t, x)$ is the penalized (Ginzburg-Landau) approximation to the unit-length director field, representing the orientation parameter of the nematic liquid-crystal.

The parameter $\epsilon>0$ is the penalization parameter, $v$ denotes the kinematic viscosity of the fluid, $\lambda$ is an elastic constant, and $\gamma$ is the relaxationtime parameter.

The coupling term $\operatorname{Div}\left(\nabla d^{T} \cdot \nabla d\right)$ preserves the regularity of the Navier-Stokes equations: when velocity $u=0$, Eq.(1.1a) becomes $\operatorname{Div}\left(\nabla d^{T}\right.$. $\nabla d)=-\operatorname{grad} p$, so that when $d_{t}=0, d$ is a solution of the static portion of Eq. (1.1c) together with the constraint $\operatorname{Div}\left(\nabla d^{T} \cdot \nabla d\right)=-\operatorname{grad} p$. This constraint pushes the gradient flow towards a "regular" $\left(H^{s}, s\right.$ sufficiently large) stationary solution. Even though the coupling term has two derivatives, analytically, it is essentially identical to the advection term $\nabla_{u} u$.

\section{Results}

We begin by extending the simplified Ericksen-Leslie model of F.H. Lin to a compact Riemannian manifold with boundary. This extension introduces a new curvature term in the basic energy laws, and provides a covariant (coordinate-independent) description of the liquid crystal dynamics. Motion on the sphere is an important application.

In Section 2, we prove that the system of Eq. (1.1) actually arises from a simple variational principle (the system was originally derived using balance laws). The variational principle is the key to our analysis, for it gives the correct scaling; namely, it shows that when $d$ is taken to have one derivative greater regularity than $u$, the liquid crystal system behaves just as the usual Navier-Stokes equations, a fact which was not previously known (see Ref. [16]). In fact, according to Ref. [19], because the interaction term $\operatorname{Div}\left(\nabla d^{T} \cdot \nabla d\right)$ in Eq. (1.1a) formally has as many derivatives as the diffusion term, the standard Galerkin procedure for obtaining local solutions, in which both the velocity and director field were simultaneously projected onto a finite dimensional subspace, had failed in prior attempts.

Section 3 is devoted to a brief review of the Riemannian geometry of the two-sphere $\mathbb{S}^{2}$ with the standard induced metric, and a computation of the covariant derivative and curvature tensor in spherical coordinates.

In Section 4, we give a very simple proof of local well-posedness of the system Eq. (1.1) on $(M, g)$ (in Theorem 4.1) using the contraction mapping 
theorem; this significantly simplifies the clever, but lengthy, modified Galerkin procedure employed by Lin and Liu in Ref. [16]. Moreover, the proof does not require use of either the maximum principle or higher-order energy laws.

In Section 5, we describe the basic energy laws on Riemannian manifolds, and show (in Propositions 5.1, 5.2, and 5.3) that on two-dimensional Riemannian manifolds with smooth boundary (possibly empty), there exists uniform estimates for $u(t, \cdot)$ in $H^{1}$ and $d(t, \cdot)$ in $H^{2}$ for almost all $t$.

In Section 6, we prove the global well-posedness ofthe system (1.1), as well as the existence of absorbing sets for $u$ in $H^{s+1}$ and $d$ in $H^{s+2}$, and hence of a compact global attractor when the dimension is $n=2$ (see Theorems 6.1 and 6.2). We then prove in Proposition 6.1 that global solutions exist in $n$ dimensions, provided that the initial data is taken sufficiently small. When $\partial M=\emptyset$, brute-force energy estimates may be computed, but when $\partial M$ is not empty, we use the Ladyzhenskaya method to obtain the uniform bounds. We remark that the existence of global attractors for this system was not previously known. We also remark that since the Navier-Stokes equations are a subsystem of Eq. (1.1), one does not expect to be able to prove results in dimension three which do not already exist for the Navier-Stokes equations; namely, the problem of unique classical solutions remains open, while weak solutions exist. ${ }^{[16]}$.

Finally, in Section 7, we introduce the Lagrangian averaged liquid crystal Eq. (7.6). This system is based on the Lagrangian averaged Navier-Stokes equations (see Ref. [22] and references therein), and is derived by averaging the Navier-Stokes flow over small spatial scales which are smaller than some positive small number $\alpha$. We show that this averaged system retains the structure of the original system derived by Lin in the form of averaged energy laws, but has the advantage of being globally well-posed on threedimensional domains (see Theorem 7.1). The averaged energy law shows that when both the fluid flow is averaged together with the director field, both $u$ and $d$ scale similarly, and $d$ is not required to have one-derivative greater regularity. Of course, physically, it seems much more natural to average the fluid flow, since the molecular orientation is already an averaged quantity. We believe that the averaged liquid crystal system will be an ideal model for numerical computation.

\section{Some Notation and Interpolation Inequalities}

We shall use the notation $H^{s}(T M)$ to denote the $H^{s}$-class vector fields on the manifold $M$. The $H^{s}(T M)$ inner-product is given, in any 
local chart, by

$$
\langle u, v\rangle_{s}=\sum_{|\alpha|=0}^{s}\left\langle D^{\alpha} u, D^{\alpha} v\right\rangle
$$

where

$$
\langle u, v\rangle=\int_{M} g(x)(u(x), v(x)) \mu(x)
$$

denotes the $L^{2}$ inner-product, $\alpha=\left(\alpha_{1}, \ldots, \alpha_{n}\right)$ is a multi-index, and

$$
|\alpha|=\alpha_{1}+\cdots+\alpha_{n}, D^{\alpha}=\partial_{1}^{\alpha_{1}} \cdots \partial_{n}^{\alpha_{n}} .
$$

Alternatively, we may choose a finite collection of vector fields $\left\{X_{j}\right\}$ spanning each tangent space $T_{x} M$, and let $D^{\alpha}=\nabla_{X_{\alpha_{1}}} \cdots \nabla_{X_{\alpha_{l}}}$ denote a differential operator of order $k=|\alpha|$. We shall denote the $H^{s}(T M)$ norm by

$$
|u|_{s}=\langle u, u\rangle_{s},
$$

$\langle\cdot, \cdot\rangle=\langle\cdot, \cdot\rangle_{0}$, and $|\cdot|=|\cdot|_{0}$. We set $H_{0}^{1}(T M)$ to consist of those vector fields in $H^{1}(T M)$ which have zero trace on $\partial M$. Similarly, vectors in $H_{h}^{1}(T M)$ have trace $h$ on $\partial M$. For $s \geq 1$ and $h \in H^{s-(1 / 2)}(T \partial M)$, we let

$$
H_{h}^{s}(T M)=\left\{d \in H^{s}(T M) \cap H^{s-(1 / 2)}(T \partial M)|d|_{\partial M}=h,|h(x)|=1\right\},
$$

denote the space of $H^{s}$ vector fields on $M$ which have $\left(H^{1}\right)$ trace $h$ on $\partial M$, where $|h(x)|^{2}=g(x)(h(x), h(x))$.

For each $x \in M$, we let $B_{x}^{\delta}=\left\{v \in T_{x} M \mid g(x)(v, v) \leq \delta\right\}$, and set $B^{\delta}=$ $\cup_{x \in M} B_{x}^{\delta}$. We let $H^{s}\left(M, B^{\delta}\right)$ denote the $H^{s}$-class maps from $M$ into $B^{\delta}$.

We have the product rule

$$
D^{\alpha}(f g)=\sum_{\substack{|\beta| \leq|| \alpha) \\ \alpha-\beta>0}} c_{\alpha, \beta}\left(D^{\beta} f\right)\left(D^{\alpha-\beta} g\right)
$$

For any integer $s \geq 0$, we set

$$
D^{s} u=\left\{D^{\alpha} u:|\alpha|=s\right\}, \quad\left\|D^{s} u\right\|_{L^{p}}=\sum_{|\alpha|=s}\left\|D^{\alpha} u\right\|_{L^{p}} .
$$

We define the spaces

$$
\begin{aligned}
\mathcal{V} & =\left\{u \in C^{\infty}(T M) \mid \operatorname{div} u=0, \quad g(u, n)=0 \quad \text { on } \partial M\right\} \\
\mathcal{W} & =\left\{u \in C_{0}^{\infty}(T M) \mid \operatorname{div} u=0\right\},
\end{aligned}
$$


and through-out the paper, we shall use $W^{s}$ and $V^{s}$ to denote the closure in $H^{s}$ of $\mathcal{V}$ and $W$, respectively. It follows that

$$
\begin{aligned}
V^{s} & =\left\{u \in H^{s}(T M)|\operatorname{div} u=0, \quad g(u, n)|_{\partial M}=0\right\}, \\
W^{s} & =\left\{u \in H^{s}(T M) \cup H_{0}^{1}(T M) \mid \operatorname{div} u=0\right\} .
\end{aligned}
$$

In section 4, we shall give an equivalent definition of $W^{s}$ using powers of the Stokes operator.

We shall need some standard interpolation inequalities, which follow from the Gagliardo-Nirenberg inequalities: ${ }^{[25,29]}$

Suppose

$$
\frac{1}{p}=\frac{i}{n}+a\left(\frac{1}{r}-\frac{m}{n}\right)+(1-a) \frac{1}{q}
$$

where $i / m \leq a \leq 1$ (if $m-i-n / r$ is an integer $\geq 1$, only $a<1$ is allowed ). Then for $f: M \rightarrow T M$,

$$
\left|D^{i} f\right|_{L^{p}} \leq C\left|D^{m} f\right|_{L^{r}}^{a} \cdot|f|_{L^{q}}^{1-a}
$$

In what follows, we shall use $C$ as a generic constant. Some specific cases in two dimensions $(n=2)$ that we shall need are as follows:

$$
\begin{aligned}
|v|_{L^{\infty}} & \leq C\left|D^{2} v\right|_{L^{2}}^{1 / 2}|v|_{L^{2}}^{1 / 2} \\
|v|_{L^{4}} & \leq C|D v|_{L^{2}}^{1 / 2}|v|_{L^{2}}^{1 / 2} \\
\left|D^{i} v\right|_{L^{2}} & \leq C|v| 1-(i / m)\left|D_{L^{2}}^{m} v\right|_{L^{2}}^{i / m} .
\end{aligned}
$$

Equation (1.3) is often called the Agmon inequality, while Eqs. (1.4)(1.5) are often referred to as the Ladyzhenskaya inequalities.

We will make use of

Lemma 1.1. (The Uniform Gronwall Lemma). Let $g, h, y, y^{\prime}$, be in $L_{\mathrm{loc}}^{1}\left(\left(t_{0}, \infty\right)\right)$ and satisfy $y^{\prime} \leq$ gy $+h$ for $t \geq t_{0}$ and

$$
\int_{t}^{t+r} g(s) d s \leq a_{1}, \quad \int_{t}^{t+r} h(s) d s \leq a_{2}, \quad \int_{t}^{t+r} y(s) d s \leq a_{3},
$$

for $t \geq t_{0}$, where $r, a_{1}, a_{2}, a_{3}$ are positive constants. Then

$$
y(t+r) \leq\left(\frac{a_{3}}{r}+a+2\right) e^{a_{1}}, \quad \forall t \geq t_{0} .
$$

See Ref. [28] for the proof.

We shall use div for the divergence operator on vector fields, and Div for the divergence operator on sections of $T^{*} M \otimes T M$. 


\section{THE VARIATIONAL PRINCIPLE}

In this section, we shall explain how the system of Eq. (1.1) arise from a simple variational principle, Eq. (1.1a) being the first variation of the action with respect to the Lagrangian flow variable, and Eq. (1.1c) being the $L^{2}$ gradient flow of the first variation of the action with respect to the director field. Previous derivations used balance arguments, rather than variational principles, to derive liquid crystal models such as Eq. (1.1).

We let $\eta(t, x)$ denote the Lagrangian flow variable, a solution of the differential equation

$$
\partial_{t} \eta(t, x)=u(t, \eta(t, x), \quad \eta(0, x)=x .
$$

For $I=[0, T]$, and each $t \in I, u \in C^{0}\left(I, W^{s}\right), s>(n / 2)+2$, the map $\eta(t, \cdot)$ : $M \rightarrow M$ is an $H^{s}$ volume-preserving diffeomorphism with $H^{s}$ inverse, and restricts to the identity map on the boundary $\partial M$. We shall denote this set of maps by $\mathcal{D}_{\mu, D}^{s}$. It is a fact, that for $s>(n / 2)+1$, the set $\mathcal{D}_{\mu, D}^{s}$ is a $C^{\infty}$ (weak) Riemannian manifold (see Ref. [8] and Ref. [26]).

We define the action function $S: \mathcal{D}_{\mu, D}^{s} H^{s+1}(T M) \cap H_{0}^{1}(T M) \rightarrow \mathbb{R}$ by

$$
\begin{aligned}
S(\eta, d)= & \frac{1}{2} \int_{I} \int_{M}\{g(\eta(x))(u(t, \eta(t, x)), u(t, \eta(t, x))) \\
& +\lambda g(\eta(x))(\nabla[d(t, \eta(t, x))], \nabla[d(t, \eta(t, x))])+2 F(d)\} \mu d t,
\end{aligned}
$$

where $F(d)=\left(1 / 4 \epsilon^{2}\right)\left(|d|^{2}-1\right)^{2}$. Notice that

$$
f(d)=\operatorname{grad} F(d),
$$

where

$$
f(d) \equiv \frac{1}{\epsilon^{2}}\left(|d|^{2}-1\right) d
$$

is the (GL) nonlinearity in Eq. (1.1c). The first term on the right-hand-side of Eq. (2.1) is the kinetic energy of the fluid, the second term is the elastic energy of the polymers, and the third term is the unit-length constraint on the director field $d$. As a consequence of the right-invariance of $S$ with respect to the lifted action of $\mathcal{D}_{\mu, D}^{s}$, we may compute the kinetic energy of the fluid as well as the elastic energy along the particle trajectory $\eta(t, x)$. The interaction, or coupling, between the velocity $u$ and the director $d$ comes precisely from the elastic energy being computed along the Lagrangian flow $\eta(t, x)$.

The elastic energy $(1 / 2) \int_{M}|\nabla d|^{2} \mu$ is a simplified form of the OseenFrank energy, given upto the null-Lagrangian by

$$
\int_{M}\left[\kappa_{1}|\operatorname{div} d|^{2}+\kappa_{2}|d \times \operatorname{curl} d|^{2}+\kappa_{3}|d \cdot \operatorname{curl} d|^{2}\right] \mu .
$$


The terms in the integrand represent, respectively, the energy due to splay, bending, and twisting of the polymers in the nematic liquid crystal. When $\kappa=$ $\kappa_{1}=\kappa_{2}=\kappa_{3}$, then (2.2) reduces to $\kappa \int_{M}|\nabla d|^{2} \mu$. We see that in the Eulerian frame, for a director field which is exactly taking values in the unit sphere, the energy is given by

$$
\text { Energy }=\frac{1}{2} \int_{M}\left(|u(x)|^{2}+\lambda|\nabla d|^{2}\right) \mu .
$$

The penalized form of this energy is then

$$
E=\frac{1}{2} \int_{M}\left(|u(x)|^{2}+\lambda|\nabla d|^{2}+2 \lambda F(d)\right) \mu
$$

where we suppress the explicit dependence on the small parameter $\epsilon>0$.

The action Eq. (2.1) is the right-translated time-integral of the energy function Eq. (2.3). The penalization was motivated by the study of harmonic maps of simply-connected domains $\Omega$ into spheres (see Ref. [2,5]); in particular, the space $H_{h}^{1}\left(\Omega, \mathbb{S}^{1}\right)=\emptyset$ when $|\operatorname{degree}(h)| \geq 1$ so that only infinite energy minimizers exist. As a fix for this problem, the penalization method is invoked, which enlarges the space of potential minimizers to $H_{h}^{1}\left(\Omega, \mathbb{R}^{2}\right)$ (which is obviously not empty) and simultaneously imposes the unit-length constraint.

To compute the first variation of $S$ with respect to $\eta$, we let $\varepsilon \mapsto \phi_{\varepsilon}$ be a smooth curve in $\mathcal{D}_{\mu, D}^{s}$ such that $\phi_{0}=e$, and $\left.(d / d \varepsilon)\right|_{\varepsilon=0} \phi_{\varepsilon}=w \in W^{s}$. We let $D / d \varepsilon$ denote the covariant derivative along the curve $\phi_{\varepsilon}$. Let $\eta^{\varepsilon}=\eta \circ \phi^{\varepsilon}$ so that

$$
\eta^{0}=\eta, \quad \text { and }\left.(d / d \varepsilon)\right|_{\varepsilon=0} \eta^{\varepsilon}=w \circ \eta .
$$

Then (setting $\lambda=1$ for the moment),

$$
\begin{aligned}
& \left\langle D_{1} S(\eta, d), \delta \eta\right\rangle \\
& =\left.\frac{d}{d \varepsilon}\right|_{\varepsilon=0} S\left(\eta^{\varepsilon}, d\right)=\int_{I} \int_{M}\left\{g(\eta(t, x))\left((D / d \varepsilon)_{\varepsilon=0} \partial_{t} \eta^{\varepsilon}(t, x), \partial_{t} \eta(t, x)\right)\right. \\
& \quad+g(\eta(t, x))\left((D / d \varepsilon)_{\varepsilon=0} \nabla\left(d\left(t, \phi^{\varepsilon}(\eta(t, x))\right), \nabla(d(t, \eta(t, x)))\right\} d x d t,\right.
\end{aligned}
$$

where $\nabla$ is computed with respect to the moving Lagrangian coordinate $y=$ $\eta(t, x)$, and where we have used $d x$ to denote the Riemannian volume-form $\mu$. We use $D_{1}$ and $D_{2}$ to denote the Frechét derivatives of $S$ with respect to $\eta$ and $d$, respectively. Integrating by parts, and using the fact that $\partial_{t} \eta=u \circ \eta$ and that $\eta$ has Jacobian determinant equal to one, we see that

$$
\begin{aligned}
& \left\langle D_{1} S(\eta, d), \delta \eta\right\rangle \\
& \quad=\int_{I} \int_{M} g(\eta(t, x))\left(-\left((D / d t) \partial_{t} \eta(t, x), w(t, \eta(t, x)) d x d t\right.\right.
\end{aligned}
$$




$$
\begin{aligned}
& +\int_{I} \int_{M} g(y)\left(\left.(D / d \varepsilon)\right|_{\varepsilon=0} \nabla d \circ \phi_{\varepsilon}(y) \cdot D \phi_{\varepsilon}(y), \nabla d(t, y)\right) d y d t \\
= & \int_{I} \int_{M} g(y)\left(\left(-u_{t}(t, y)-\nabla_{u} u(t, y)-\operatorname{grad} p(t, y), w(t, y)\right) d y d t\right. \\
& +\int_{I} \int_{M}\left\{g(y)\left(\nabla_{w}(\nabla d), \nabla d\right)+g(y)(\nabla d(t, y) \cdot \nabla w, \nabla d)\right\} d y d t \\
= & \int_{I} \int_{M} g(y)\left(\left(-u_{t}(t, y)-\nabla_{u} u(t, y)-\operatorname{grad} p(t, y), w(t, y)\right) d y d t\right. \\
& +\int_{I} \int_{M} g(y)\left(-\operatorname{Div}\left(\nabla d^{T} \cdot \nabla d\right), w\right) d y d t,
\end{aligned}
$$

where $D \phi_{\varepsilon}$ denote the matrix of partial derivatives of $\phi_{\varepsilon}$, and where the last equality follows from the fact that $\left\langle\nabla_{w}(\nabla d), \nabla d\right\rangle=0$, since $\operatorname{div} w=0$. Thus, since $w$ is an arbitrary variation of $\eta$, we arrive at the Euler-Lagrange equation

$$
u_{t}+\nabla_{u} u=-\operatorname{grad} p-\operatorname{Div}\left(\nabla d^{T} \cdot \nabla d\right) .
$$

The viscosity (diffusion) term follows from the Itö formula by allowing $\eta(t, x)$ to be a stochastic process, and replacing deterministic time derivatives with stochastic backward-in-time mean derivatives (see Ref. [12]). Thus Eq. (1.1a) follows as the first variation of the action function $S$ with respect to $\eta$. Eq. (1.1b) follows immediately from the fact that $\eta$ is volume-preserving.

Letting $d^{\varepsilon}=d+\varepsilon \delta d$, a much simpler computation verifies that

$$
\left\langle D_{2} S(\eta, d), \delta d\right\rangle=\left.\frac{d}{d \varepsilon}\right|_{\varepsilon=0} S\left(\eta, d^{\varepsilon}\right)=\int_{I} \int_{M} g(y)(\hat{\Delta} d-f(d), \delta d) d y d t,
$$

where

$$
\hat{\Delta} d=-\nabla^{*} \nabla
$$

is the rough Laplacian and $\nabla^{*}$ is the $L^{2}$ formal adjoint of the covariant derivative $\nabla$. Hence, Eq. (1.1c) is simply the $L^{2}$ gradient flow of $d \mapsto$ $S(\eta, d)$ given by

$$
\frac{d}{d t}\left(d(t, \eta(t, x))=D_{2} S(\eta, d)=\hat{\Delta} d-f(d) .\right.
$$

We remark that

$$
\operatorname{Div}\left(\nabla d^{T} \cdot \nabla d\right)=\nabla d^{T} \cdot \hat{\Delta} d+g\left(R\left(e_{i}, \cdot\right) d, \nabla_{e_{i}} d\right),
$$

where $R$ is the Riemannian curvature tensor which is defined for vector fields $X, Y, Z$ on $M$ by

$$
R(X, Y) Z=\nabla_{X} \nabla_{Y} Z-\nabla_{Y} \nabla_{X} Z+\nabla_{[X, Y]} Z,
$$


and where $\left\{e_{i}\right\}$ is any local orthonormal frame. The curvature term in Eq. (2.5) will play an important role in the energy behavior of the system.

\section{LIQUID CRYSTALS ON THE SPHERE}

Let $\left(y^{1}, y^{2}, y^{3}\right)$ be the standard Cartesian coordinates on $\mathbb{R}^{3}$, and set

$$
y^{1}=\sin \theta \cos \phi, \quad y^{2}=\sin \theta \sin \phi, \quad y^{3}=\cos \theta .
$$

Letting $x^{1}=\theta$ and $x^{2}=\phi$ denote spherical coordinates on $\mathbb{S}^{2}$, the induced metric (from $\mathbb{R}^{3}$ ) is given by

$$
g=d \theta^{2}+\sin ^{2} \theta d \phi^{2},
$$

and the induced volume form is

$$
\mu=\sqrt{\operatorname{det} g} d \theta d \phi=\sin \theta d \theta d \phi .
$$

For a vector field $v$ on $\mathbb{S}^{2}$, we may express it as

$$
v=v_{\theta} e_{\theta}+v_{\phi} e_{\phi},
$$

where $\left(e_{\theta}=\partial / \partial \theta, e_{\phi}=(1 / \sin \theta)(\partial / \partial \phi)\right)$ is the usual orthonormal basis.

Given two vector fields $v$ and $w$ on $\mathbb{S}^{2}$, the covariant derivative of $v$ in the direction $w$ is given by

$$
\begin{aligned}
\nabla_{w} v= & \left(w_{\theta} \frac{\partial v_{\theta}}{\partial \theta}+\frac{w_{\varphi}}{\sin \theta} \frac{\partial v_{\theta}}{\partial \varphi}-w_{\varphi} v_{\varphi} \cot \theta\right) e_{\theta} \\
& +\left(w_{\theta} \frac{\partial v_{\varphi}}{\partial \theta}+\frac{w_{\varphi}}{\sin \theta} \frac{\partial v_{\varphi}}{\partial \varphi}+w_{\varphi} v_{\theta} \cot \theta\right) e_{\varphi} \\
= & \left(w^{\theta} \frac{\partial v^{\theta}}{\partial \theta}+w^{\varphi} \frac{\partial v^{\theta}}{\partial \varphi}-w^{\varphi} v^{\varphi} \sin \theta \cos \theta\right) \frac{\partial}{\partial \theta} \\
& +\left(w^{\theta} \frac{\partial v^{\varphi}}{\partial \theta}+w^{\varphi} \frac{\partial v^{\varphi}}{\partial \varphi}+\left(w^{\theta} v^{\varphi}+w^{\varphi} v^{\theta}\right) \cot \theta\right) \frac{\partial}{\partial \varphi}
\end{aligned}
$$

We component notation, $(\nabla v)_{j}^{i}=\nabla_{j} v^{i}$.

The (rate of) deformation tensor is computed simply by symmetrization of the matrix $\nabla u$. The divergence of a vector field $v$ on $\mathbb{S}^{2}$ is given by

$$
\operatorname{div} v=\frac{1}{\sin \theta} \frac{\partial}{\partial \theta}\left(\sin \theta v_{\theta}\right)+\frac{1}{\sin \theta} \frac{\partial v_{\phi}}{\partial \phi},
$$


and the gradient of a function $p: \mathbb{S}^{2} \rightarrow \mathbb{R}$ is

$$
\operatorname{grad} p=\frac{\partial p}{\partial \theta} e_{\theta}+\frac{1}{\sin \theta} \frac{\partial p}{\partial \phi} e_{\phi} .
$$

In coordinates,

$$
[\text { Div Def } u]^{i}=\frac{1}{2} \nabla_{j}\left[(\nabla u)_{j}^{i}+(\nabla u)_{i}^{j}\right]
$$

and the rough Laplacian of a vector field $d$ is given by

$$
[\hat{\Delta} d]^{i}=g^{j k} \nabla_{k} \nabla_{j} d^{i}
$$

where $g^{j k}$ denotes the components of $g^{-1}$. As to the energy law on $\mathbb{S}^{2}$, we must specify all of the components of the Riemannian curvature tensor $R$. Because of the symmetries of $R$, each component $R_{i j k l}$ is either 0 or plus-orminus

$$
R_{1212}=R_{2121}=\sin ^{2} \theta
$$

We have thus shown how to explicitly compute each term of Eq.(1.1) in the case that $M=\mathbb{S}^{2}$ with the induced metric.

Because $\chi\left(\mathbb{S}^{2}\right)$ does not vanish, the seminorm

$$
\int_{\mathbb{S}^{2}}|\nabla u(x)|^{2} \mu, \quad u \in H^{1}\left(T \mathbb{S}^{2}\right)
$$

induces an $H^{1}$ norm which is equivalent to the usual norm $|u|_{1}$.

\section{LOCAL WELL-POSEDNESS}

Let $P$ denote the Leray orthogonal projection from $L^{2}(T M)$ onto $W^{0}$, and let

$$
A=-P \text { Div Def }
$$

denote the Stokes operator, an unbounded, positive, self-adjoint operator on $W^{0}$, with domain $D(A)=H^{2}(T M) \cap W^{1}$. As usual, we set

$$
W^{s}=D\left(A^{s / 2}\right), \quad s \geq 0 .
$$

This is a Hilbert space with inner-product $\left\langle A^{s / 2} u, A^{s / 2} v\right\rangle$ for $u, v \in D\left(A^{s / 2}\right)$. The norm $\left|A^{s / 2} u\right|$ is equivalent to the $H^{s}$ norm. 
We first prove the local well-posedness of classical solutions. Let

$$
\dot{H}_{h}^{s+1}(T M):=\left\{d \in H_{h}^{s+1}(T M) \mid \hat{\Delta} d=0 \quad \text { on } \partial M\right\}
$$

for vector fields $h$ defined on the boundary $\partial M$.

Theorem 4.1. Suppose $2 \leq \operatorname{dim}(M) \leq 4$ and set $s_{0}=(n / 4)+(1 / 2)$. For $s \in\left(s_{0}, 5 / 2\right)$ and $u_{0} \in W^{s}, d_{0} \in \dot{H}_{h}^{s+1}(T M)$, there exists $T>0$ depending only on the data and $M$, such that

$$
u \in C^{0}\left([0, T], W^{s}\right), \quad d \in C^{0}\left([0, T], \dot{H}_{h}^{s+1}(T M)\right)
$$

are solutions to the system of Eq. (1.1).

Proof. It will be convenient to recast the Eqs. (1.1c) and (1.1e) so that the solution has zero trace on $\partial M$. For any boundary data $h \in H^{s+(1 / 2)}(T \partial M)$, we may choose $\psi \in H^{s+1}(T M)$ such that $\operatorname{trace}(\psi)=h$ and $\hat{\Delta} \psi=0$ on $\partial M$. Let

$$
\tilde{d}=d-\psi, \quad \text { so that }\left.\tilde{d}\right|_{\partial M}=0 \quad \text { and }\left.\hat{\Delta} \tilde{d}\right|_{\partial M}=0 .
$$

We rewrite the system (1.1) as an evolution equation in

$$
\begin{aligned}
X^{s} \equiv W^{s} \oplus \dot{H}_{0}^{s+1}(T M): & \\
u_{t}+v A u+P \nabla_{u} u & =-P \lambda \operatorname{Div}\left(\nabla[\tilde{d}+\psi]^{T} \cdot \nabla[\tilde{d}+\psi]\right), \\
\tilde{d}_{t}+\nabla_{u} \tilde{d} & =\gamma(\hat{\Delta} d-\tilde{f}(\tilde{d})+\hat{\Delta} \psi), \\
u & =0 \quad \text { on } \partial M, \tilde{d}=0 \quad \text { on } \partial M \quad \text { or } \partial M=\emptyset, \\
u(0, x) & =u_{0}, \tilde{d}(0, x)=\tilde{d}_{0}(x) \equiv d_{0}(x)+\psi(x),
\end{aligned}
$$

where

$$
\tilde{f}(\tilde{d}) \equiv \frac{1}{\epsilon^{2}}\left(|\tilde{d}+\psi|^{2}-1\right)(\tilde{d}+\psi) .
$$

We define the vector

$$
x \equiv(u, \tilde{d}) \in X^{s} ;
$$

and define the maps

$$
\begin{aligned}
& \phi_{1}: X^{s} \rightarrow Y_{1}, \\
& \phi_{1}(x)=-P\left(\nabla_{u} u+\operatorname{Div}\left(\nabla[\tilde{d}+\psi]^{T} \cdot \nabla[\tilde{d}+\psi]\right)\right),
\end{aligned}
$$


and

$$
\begin{aligned}
& \phi_{2}: X^{s} \rightarrow Y_{2}, \\
& \phi_{2}(x)=-\nabla_{u} \tilde{d}-\gamma \tilde{f}(\tilde{d})+\gamma \hat{\Delta} \psi,
\end{aligned}
$$

for some Banach spaces $Y_{1}$ and $Y_{2}$ to be determined.Thus, the vector

$$
\Phi \equiv\left(\phi_{1}, \phi_{2}\right): X^{s} \rightarrow Y_{1} \times Y_{2} .
$$

Next, we define the semigroup

$$
S(t)=\left[\begin{array}{cc}
e^{-t \nu A} & 0 \\
0 & e^{t \gamma \hat{\Delta}}
\end{array}\right]
$$

We can now express the system Eq. (4.1) as the integral equation

$$
x(t, \cdot)=S(t) x_{0}-\int_{0}^{t} S(t-s) \Phi(x(s)) d s=\Psi x(t, \cdot) .
$$

Since for $s \in\left(s_{0}, 5 / 2\right), e^{-t v A}: W^{s} \rightarrow W^{s}$ and $e^{t \gamma \hat{\Delta}}: \dot{H}_{0}^{s+1}(T M) \rightarrow$ $\dot{H}_{0}^{s+1}(T M)$ are strongly continuous semigroups, it follows that

$$
S(t): X^{s} \rightarrow X^{s} \text { is a strongly continuous semigroup for } t \geq 0,
$$

and that for $t>0, S(t): \mathcal{V}^{s-1} \times H^{s}(T M) \rightarrow X^{s}$. For $s \in\left(s_{0}, 2\right)$, we set

$$
Y_{1}=V^{0}, \quad Y_{2}=H^{1}(T M) ;
$$

we have the standard estimate (see Ref. [29]) that

$$
\|S(t)\|_{L\left(Y_{1} \times Y_{2}, X^{s}\right)} \leq C t^{-\gamma}, \quad \gamma \in(0,1), \quad t \in(0,1] .
$$

Thus, it suffices to prove that for $s \in\left(s_{0}, 2\right)$, the map $\Phi: X^{s} \rightarrow Y_{1} \times Y_{2}$ is locally Lipschitz.

Using Lemma 5.3, Ref. [29, Chapter 17], we have that for $s \in\left(s_{0}, 2\right)$ $(f, g) \mapsto f g: H^{s} \times H^{s} \rightarrow H^{1}$. It follows that $u \mapsto u \otimes u: H^{s} \rightarrow H^{1}$, $d \mapsto\left(\nabla d^{T} \cdot \nabla d\right): H^{s+1} \rightarrow H^{1}$, and $(u, d) \mapsto \nabla_{u} d: H^{s} \times H^{s+1} \rightarrow H^{1}$. The fact that $d \mapsto f(d): H^{s+1} \rightarrow H^{1}$ follows because $H^{s+1}$ forms a Schauder ring. This, together with the fact that $P: H^{s}(T M) \rightarrow V^{s}$ is a bounded projection, yields that

$$
\Phi: X^{s} \rightarrow Y_{1} \times Y_{2} \text { is a locally Lipschitz map. }
$$

Now for the interval of values of $2 \leq s<5 / 2$, we proceed as follows. We set

$$
Y_{1}=V^{\sigma}, \quad Y_{2}=H^{1+\sigma}(T M), \quad \sigma \in\left[0, \frac{1}{2}\right) .
$$


In this case, we consider the space $X^{s}$ with $s-\sigma \in(0,2)$ and find that Eq. (4.6) still holds. Furthermore, Eq. (4.7) will continue to hold provided that we find the interval of values of $s$ for which the multiplication operation $(f, g) \mapsto f g$ maps $H^{s} \times H^{s}$ into $H^{1+\sigma}$. Lemma 5.7 of Ref. [29] shows that for $\delta \in(0,1 / 2)$ taken sufficiently small, and $\sigma=1 / 2-\delta$, there exists $\bar{s} \in(\sigma, 2+\sigma)$ such that this multiplication operation holds.

Fix $\alpha>0$ and set

$$
Z=\left\{x \in C\left([0, T], X^{s}\right) \mid x(0)=\left(u_{0}, \tilde{d}_{0}\right), \quad\|x(t, \cdot)-x(0)\|_{X^{s}}<\alpha\right\} .
$$

We want to choose $T$ sufficiently small so that $\Psi: Z \rightarrow Z$ is a contraction. By Eq. (4.5), we can choose $T_{1}$ so that

$$
\left\|S(t) x_{0}-x_{0}\right\|_{X^{s}} \leq \alpha / 2 \quad \forall t \in\left[0, T_{1}\right] .
$$

If $x \in Z$, then by (4.7) we have a bound

$$
\|\Phi(x(s))\|_{\mathcal{V}^{s-1} \times H^{s}(T M)} \leq K_{1} \quad \text { for } s \in\left[0, T_{1}\right] .
$$

Using (4.6), we have that

$$
\left\|\int_{0}^{t} S(t-s) \Phi(x(s)) d s\right\|_{X^{s}} \leq C t^{1 / 2} K_{1}
$$

hence, for $t \in\left[0, T_{2}\right]$, and with $x=(u, \tilde{d})$,

$$
\begin{aligned}
& \|\Psi(u(t), \tilde{d}(t))-\Psi(v(t), e(t))\|_{X^{s}} \\
& \quad=\left\|\int_{0}^{t} S(t-s)[\Phi(u(s), \tilde{d}(s))-\Phi(v(s), e(s))] d s\right\|_{X^{s}} \\
& \quad \leq C t^{1 / 2} K \sup \|(u(s), \tilde{d}(s))-(v(s), e(s))\|_{X^{s}} .
\end{aligned}
$$

Choosing $T \leq T_{2}$ small enough so that $C T^{1 / 2} K<1$, we see that by the contraction mapping theorem, $\Psi$ has a unique fixed point in $Z$, and this proves the theorem.

Remark 4.1. In the case that $\partial M=\emptyset$, we may take $s>s_{0}$ and place no restrictions on the dimension $n$ of the manifold. This difference arises because $e^{t \Delta}$ is a strongly continuous semi-group in all $H^{s}, s>s_{0}$, when there is no boundary. In the case that $\partial M \neq \emptyset$, we used the subspace 
$\dot{H}_{0}^{s+1}$ to obtain a strongly continuous semigroup for $s \in\left(s_{0}, 5 / 2\right)$. In order to obtain strong continuity at $t=0$ for larger values of $s$, it is necessary to insist upon the additional compatibility conditions, $\Delta^{2} d_{0}=\cdots \Delta^{m} d_{0}=0$ on $\partial M$ for all integers $2 \leq m<s / 2$.

\section{BASIC ENERGY LAWS ON RIEMANNIAN MANIFOLDS}

In this section, we shall establish a priori estimates for solutions of the liquid crystal system Eq. (1.1) which are uniform in time. By the local wellposedness result, Theorem 4.1, we may assume that there exists unique solutions $u \in C^{0}\left([0, T], W^{s}\right)$ and $d \in C^{0}\left([0, T], \dot{H}_{h}^{s+1}(T M)\right)$ for some time $T$. Of course, $d$ is also in $C^{1}\left([0, T], \dot{H}_{h}^{s-1}(T M)\right)$. We shall state our estimates on this time interval, and use the continuation argument to extend the time interval; alternatively, one may mollify the Eq. (1.1) and obtain smooth global solutions to the mollified equations, and use the a priori estimates to pass to limits of the mollification parameter.

In this section, we show that the system Eq. (1.1) admits the following energy law:

$$
\begin{aligned}
\frac{d}{d t} E(u, d) & :=\frac{d}{d t} \frac{1}{2} \int_{M}\left(|u(x)|^{2}+\lambda|\nabla d|^{2}+2 \lambda F(d)\right) \mu \\
& =-\left(v|\operatorname{Def} u|^{2}+\lambda \gamma|\hat{\Delta} d-f(d)|^{2}\right)-\lambda \operatorname{Trace}\langle R(\cdot, u) d, \nabla . d\rangle,
\end{aligned}
$$

for $0<t \leq T$, where $e_{i}$ denotes a local orthonormal frame. When $M$ has zero curvature, then $E$ is a Lyapunov function for the system Eq. (1.1), with the property that

$$
E(u(t), d(t)) \leq E\left(u_{0}, d_{0}\right), \quad \forall t \geq 0,
$$

and if $E\left(u\left(t_{1}\right), d\left(t_{1}\right)\right)=E\left(u\left(t_{2}\right), d\left(t_{2}\right)\right)$ for $t_{1}<t_{2}$, then $(u(t), d(t))=\left(u^{*}, d^{*}\right)$ are equilibrium solutions. Even, when the curvature $R \neq 0$, the energy remains uniformly bounded.

For the following lemma, which is standard, we suppose that $x \mapsto$ $d(t, x)$ is $C^{2}$, and that $t \mapsto d(t, x)$ is $C^{1}$. By Sobolev's embedding theorem, this is accomplished with $s>2$.

Lemma 5.1. Suppose that $\partial M \neq \emptyset$,

$$
|d(t, \cdot)|_{L^{\infty}} \leq\left|d_{0}\right|_{L^{\infty}}, \quad \forall t \in[0, T] .
$$

Proof. For any $v$, we will use the notation $|v(x)|^{2}=g(x)(v(x), v(x))$. We compute the pointwise inner-product of Eq. (5.3b) with $d$, and use the fact 
that $g(\hat{\Delta} d(x), d(x))=(1 / 2) \Delta|d(x)|^{2}-|\nabla d(x)|^{2}$. Hence, $\forall x \in M$, we obtain

$$
\left.\frac{1 d}{2 d t}|d|^{2}+\frac{1}{2} g\left(\operatorname{grad}|d|^{2}, u\right)\right)-\frac{1}{2} \Delta|d|^{2}+|\nabla d|^{2}=-\frac{1}{\epsilon^{2}}\left[|d|^{4}-|d|^{2}\right] .
$$

We consider $(x, t) \in Q \equiv \bar{M} \times[0, T]$; since $|d(t, x)|$ is regular, it has a maximum on this set. Assume that $|d(t, x)|^{2} \geq\left|d_{0}\right|_{L^{\infty}}^{2} \geq 1$, and that a maximum occurs in $Q$. Then at this point, $\operatorname{grad}|d|^{2}=0$, and $\Delta|d|^{2}<0$, so that by Eq. (5.2) $(d / d t)|d|^{2}<0$, but this is a contradiction.

Thus, for $d_{0} \in H^{s+1}\left(M, B^{\delta}\right), s>2,|d(t, \cdot)|_{L^{\infty}} \leq \delta, \forall t>0$.

Remark 5.1. To achieve the bound of the previous lemma, we could have replaced the term $\nabla_{u} d$ in Eq. (1.1c) by $\nabla_{u^{\theta}} d$ where $u^{\theta}=J^{\theta} * u$ with $J^{\theta}$ a Friedrichs mollifier. Since $u^{\theta}$ is smooth, standard parabolic regularity shows that $d$, which is a function of $\theta$, is smooth as a function of $(t, x)$, and hence satisfies the maximum principle. Then passing to the limit as $\theta \rightarrow 0$, and using Eq. (5.1), shows that the bound given by Lemma 5.1 holds for $s>1$ as well.

Proposition 5.1. For $t \in(0, T]$, the energy law (5.1) holds, and solutions $(u, d)$ remain bounded in $W^{0} \times H_{h}^{1}(T M)$ if $\partial M \neq \emptyset$ and in $W^{0} \times H^{1}\left(M, B^{\delta}\right)$ if $\partial M=\emptyset$.

Proof. Using the formula Eq. (2.5), we rewrite Eqs. (1.1a) and (1.1c) as

$$
\begin{aligned}
u_{t}+\nabla_{u} u= & -\operatorname{grad} p+v \text { Div Def } u-\lambda \nabla d^{T} \cdot \hat{\Delta} d \\
& -g\left(R\left(e_{i}, \cdot\right) d, \nabla_{e_{i}} d\right), \\
d_{t}+\nabla_{u} d= & \gamma\left(\hat{\Delta} d-\frac{1}{\epsilon^{2}}\left(|d|^{2}-1\right) d\right),
\end{aligned}
$$

where $e_{i}$ is any local orthonormal frame. Adding the $L^{2}$ inner-product of Eq. (5.3a) with $u$ to the $L^{2}$ inner-product of Eq. (5.3b) with $d_{t}+\nabla_{u} d$, we obtain the basic energy law

$$
\begin{aligned}
& \frac{1}{2} \frac{d}{d t}\left(|u|^{2}+\lambda|\nabla d|^{2}+2 \lambda \int_{M} F(d(x)) \mu\right) \\
& \quad=-\left(v|\operatorname{Def} u|^{2}+\lambda \gamma|\hat{\Delta} d-f(d)|^{2}\right)-\lambda \operatorname{Trace}\langle R(\cdot, u) d, \nabla . d\rangle .
\end{aligned}
$$

In the case of a flat manifold, such as a bounded domain in $\mathbb{R}^{n}, R=0$, and Eq. (5.4) reduces to the basic energy law Eq. (1.8) in Ref. [16]. 
From Lemma 5.1, we have that

$$
|d(t, \cdot)|_{L^{\infty}} \leq C, \quad t>0
$$

It follows that

$$
\begin{aligned}
\operatorname{Trace}\langle R(\cdot, u) d, \nabla . d\rangle & \leq C|R|_{L^{\infty}}|u||d|^{1 / 2}|\hat{\Delta} d|^{1 / 2} \\
& \leq C \varepsilon|\hat{\Delta} d|^{2}+\frac{C}{\varepsilon}\left(|M||R|_{L^{\infty}}|u|\right)^{4 / 3} \\
& \leq C \varepsilon|\hat{\Delta} d|^{2}+\varepsilon|u|^{2}+\frac{C}{\varepsilon^{4}}\left(|M||R|_{L^{\infty}}\right)^{4} \\
& \leq C \varepsilon|\hat{\Delta} d|^{2}+c_{0}^{-1}(M) \varepsilon|\operatorname{Def} u|^{2}+\frac{C}{\varepsilon^{4}}\left(|M||R|_{L^{\infty}}\right)^{4},
\end{aligned}
$$

where the second and third inequalities follow from Young's inequality Eq. (6.2), and the last inequality follows from the Poincare inequality for $c_{0}(M)>0$, a positive constant depending on $M$. Taking $\varepsilon>0$ sufficiently small so that

$$
K=\min \left(c_{0}-\varepsilon, 1-2 \varepsilon\right)>0,
$$

the basic energy law Eq. (5.4) on a Riemannian manifold yields the following differential inequalities:

$$
\begin{aligned}
& \frac{1}{2} \frac{d}{d t}\left[|u|^{2}+|\nabla d|^{2}+2 \int_{M} F(d) \mu\right] \\
& \quad \leq-K C\left[|\operatorname{Def} u|^{2}+|\hat{\Delta} d|^{2}+2 \int_{M} F(d) \mu\right]+\rho_{0}, \\
& \frac{1}{2} \frac{d}{d t}\left[|u|^{2}+|\nabla d|^{2}+2 \int_{M} F(d) \mu\right] \\
& \quad \leq-K C\left[|u|^{2}+|\nabla d|^{2}+2 \int_{M} F(d) \mu\right]+\rho_{0},
\end{aligned}
$$

where

$$
\rho_{0}=C\left[(K+C / \varepsilon-1)|M|+\frac{1}{\varepsilon^{4}}|M|^{4}|R|_{L^{\infty}}\right] \text {. }
$$


Using the classical Gronwall lemma, we obtain

$$
\begin{aligned}
& {\left[|u|^{2}+|\nabla d|^{2}+2 \int_{M} F(d) \mu\right]} \\
& \quad \leq\left[\left|u_{0}\right|^{2}+\left|\nabla d_{0}\right|^{2}+2 \int_{M} F\left(d_{0}\right) \mu\right] e^{-K C t}+\rho_{0}\left(1-e^{-K C t}\right) .
\end{aligned}
$$

Thus, in the case that $T=\infty$,

$$
\begin{gathered}
\limsup _{t \rightarrow \infty}\left[|u(t)|^{2}+|\nabla d(t)|^{2}+2 \int_{M} F(d(t)) \mu\right] \leq \rho_{0} \\
\limsup _{t \rightarrow \infty}\left[|u(t)|^{2}+|\nabla d(t)|^{2}\right] \leq \rho_{0}+2|M|,
\end{gathered}
$$

where $\rho_{0}$ is given by Eq. (5.7).

When $R=0$, we do not need to rely on the maximum principle to establish Proposition 5.1 or to establish the existence of an $L^{\infty}$ absorbing set for Eq. (5.3b).

Lemma 5.2. Suppose that $\partial M=\emptyset, R=0$, and that $d$ is a global solution to Eq. (1.1c). There exists $\rho_{\infty}>0$ independent of $d_{0}$ such that for sufficiently large $t$,

$$
|d(t, \cdot)|_{L^{\infty}} \leq \rho_{\infty}
$$

For solutions on $[0, T],\|d(t, \cdot)\|_{L^{\infty}}$ remains uniformly bounded.

Proof. From the energy law Eq. (5.1) with $R=0$, there is a $t>t^{*}(\bar{r})$ for which all bounded subsets of $L^{2}(T M)$, contained in the ball of radius $\bar{r}$, will enter the $L^{2}$ ball of radius $\rho_{0}$ (see Ref. [28]).

For $p>2$, we take the pointwise inner-product of Eq. (5.3b) with $p|d|^{p-2} d$ and integrate over $M$ to obtain the differential inequality

$$
\begin{aligned}
\frac{d}{d t}|d|_{L^{p}}^{p}= & -p \int_{M}|\nabla d|^{2}|d|^{p-2} \mu-p(p-2) \int_{M}|d|^{p-2}|\nabla| d||^{2} \mu \\
& +\frac{1}{\epsilon^{2}}\left(|d|_{L^{p}}^{p}-|d|_{L^{p}}^{p-2}\right) \\
\leq & -p(p-2) \int_{M}|d|^{p-2}|\nabla| d||^{2} \mu+\frac{1}{\epsilon^{2}}|d|_{L^{p}}^{p} .
\end{aligned}
$$


Using the interpolation inequality (see Ref. [20])

$$
\begin{aligned}
|d|_{L^{p}}^{p} & \leq C_{p}|d|^{2}\left(\left.\left.\int_{M}|\nabla| d\right|^{p / 2}\right|^{2} \mu\right)^{(p-2) / p} \\
& =C_{p}|d|^{2}\left(\frac{p^{2}}{4} \int_{M}|d|^{p-2}|\nabla| d \|^{2} \mu\right)^{(p-2) c / p}
\end{aligned}
$$

we see that

$$
-p(p-2) \int_{M}|d|^{p-2}|\nabla| d||^{2} \mu \leq\left[\left(\frac{1}{C_{p}}\right)^{p / 2(p-2)} \frac{4 p(p-2)}{p^{2}} \rho_{0}^{p /(p-2)}\right]\left(|d|_{L^{p}}^{p}\right)^{p /(p-2)} .
$$

Using Bernoulli's trick in the differential inequality Eq. (5.9), we get a uniform bound for $|d(t, \cdot)|_{L^{p}}$ which is independent of $p$ (even if the constant $C_{p}$ tends to infinity), and thus we may pass to the limit as $p \rightarrow \infty$.

From Lemma 5.2, we obtain

Proposition 5.2. If $R=0$ and $\partial M=\emptyset$, we have the energy law

$$
\frac{d}{d t} \frac{1}{2} \int_{M}\left(|u(x)|^{2}+\lambda|\nabla d|^{2}+2 \lambda F(d)\right) \mu=-\left(\nu|\operatorname{Def} u|^{2}+\lambda \gamma|\hat{\Delta} d-f(d)|^{2}\right),
$$

and solutions $(u, d)$ remain bounded in $W^{0} \times H^{1}(T M)$ for all $t \in(0, T]$.

In two dimensions with $R=0$, the analysis of Ref. [16] yielded global solutions of Eq. (1.1) with $u$ of class $H^{1}$ and $d$ of class $H^{2}$, but the estimates were not uniform in time.

Proposition 5.3. For $n=2, u(t, \cdot)$ is uniformly bounded in the $H^{1}$ topology and $d(t, \cdot)$ is uniformly bounded in the $H^{2}$ topology, for $t \in[0, T]$.

Proof. It follows from Eq. (5.6a) that

$$
\begin{gathered}
K C \int_{t}^{t+r}\left[|\operatorname{Def} u(s)|^{2}+|\hat{\Delta} d(s)|^{2}+2 \int_{M} F(d(s)) \mu\right] d s \\
\leq r \rho_{0}+\left[|u|^{2}+|\nabla d|^{2}+2 \int_{M} F(d) \mu\right], \quad \forall r>0,
\end{gathered}
$$


so

$$
\limsup _{t \rightarrow \infty} \int_{t}^{t+r}\left[|\operatorname{Def} u(s)|^{2}+|\hat{\Delta} d(s)|^{2}+2 \int_{M} F(d(s) \mu] d s \leq(r+1) \rho_{0} .\right.
$$

Therefore,

$$
\int_{t}^{t+r}\left[|\operatorname{Def} u(s)|^{2}+|\hat{\Delta} d(s)|^{2}+2 \int_{M} F(d(s)) \mu\right] d s \text { is uniformly bounded. }
$$

Now let

$$
A^{2}=|\operatorname{Def} u|^{2}+|\hat{\Delta} d-f(d)|^{2}, \quad B^{2}=|\nabla \operatorname{Def} u|^{2}+|\nabla(\hat{\Delta} d-f(d))|^{2} .
$$

Using Eqs. (5.10) and (5.5), we have that

$$
\int_{t}^{t+r} A^{2}(s) d s \text { is uniformly bounded. }
$$

In the case that $R=0$, it follows from a similar argument as in Eqs. (4.4)(4.8) of Ref. [16] that for some constants $c_{1}, c_{2}, c_{3}>0$,

$$
\frac{d}{d t} A^{2}(t)+c_{1} B^{2}(t) \leq c_{2} A^{4}(t)+c_{3}
$$

When $R \neq 0$, we find that for $c_{4}>0$,

$$
\frac{d}{d t} A^{2}(t)+c_{1} B^{2}(t) \leq c_{2} A^{4}(t)+c_{3}+c_{4} \operatorname{Trace}\langle R(\cdot, \Delta u) d, \nabla . d\rangle
$$

The last term is bounded by $\varepsilon|R|_{L^{\infty}}^{2} \mid$ Div Def $\left.u\right|^{2}+(C / \varepsilon)|\nabla d|^{2}$, so by taking $\varepsilon>0$ sufficiently small and adjusting the constants as necessary, we see that Eq. (5.12) still holds.

Thus, using Eq. (5.8) and appealing to the uniform Gronwall Lemma 1.1, we see that

$A(t)$ is uniformly bounded in time.

Because of Eq. (5.5), we may extract a uniform bound for $\mid$ Def $\left.u\right|^{2}+|\hat{\Delta} d|^{2}$. Hence, we have an a priori uniform bound for $u$ in the $H^{1}$ topology and for $d$ in the $H^{2}$ topology. 


\section{GLOBAL WELL-POSEDNESS AND GLOBAL ATTRACTORS}

\subsection{Manifolds Without Boundary}

We shall first consider a closed Riemannian manifold such as, for example, the two-sphere $\mathbb{S}^{2}$; for such manifolds, simple brute-force energy estimates work.

Theoem 6.1. For $n=2, s>1, \partial M=\emptyset$, and $u_{0} \in W^{s}, d_{0} \in H^{s+1}\left(M, B^{\delta}\right)$,

$$
\begin{aligned}
& u \in C^{0}\left([0, \infty), W^{s}\right) \cap C^{\infty}((0, \infty) \times M), \\
& d \in C^{0}\left([0, \infty), H^{s+1}\left(M, B^{\delta}\right)\right) \cap C^{\infty}((0, \infty) \times M)
\end{aligned}
$$

are solutions to the system of Eq. (1.1). Moreover, there exists a compact global attractor for the system Eq. (1.1) in $W^{s} \times H^{s+1}\left(M, B^{\delta}\right)$. In the case that $R=0$, we can replace $H^{s+1}\left(M, B^{\delta}\right)$ with $H^{s+1}(T M)$.

Proof. Taking the $H^{s}$ inner-product of Eq. (1.1a) with $u$ and adding the $H^{s+1}$ inner-product of Eq. (1.1c) with $d$, we find that

$$
\begin{aligned}
\frac{1}{2} \frac{d}{d t}\left(|u|_{s}^{2}+|d|_{s+1}^{2}\right) \leq & -v|u|_{s+1}^{2}-\gamma|d|_{s+2}^{2}+\lambda\left|\left\langle P \operatorname{Div}\left(\nabla d^{T} \cdot \nabla d\right), u\right\rangle_{s}\right| \\
& +\left|\left\langle P \nabla_{u} u, u\right\rangle_{s}\right|+\left|\left\langle\nabla_{u} d, d\right\rangle_{s+1}\right|+\gamma\left|\langle f(d), d\rangle_{s+1}\right| .
\end{aligned}
$$

We shall estimate each of the nonlinear terms on the right-hand-side of Eq. (6.1); as we showed in the proof of Theorem 4.1, the projection $P$ acting on the nonlinear terms, maps $H^{s-1}$ into itself continuously, so it suffices to estimate $\left\langle\nabla_{u} u, u\right\rangle_{s}$ and $\left\langle\operatorname{Div}\left(\nabla d^{T} \cdot \nabla d\right), u\right\rangle_{s}$ in the third and fourth terms. Using Proposition 5.3, we may interpolate the nonlinear terms between $|u|_{1}$ and $|u|_{s+1}$ and $|d|_{2}$ and $|d|_{s+2}$, respectively.

We have that

$$
\begin{aligned}
\left\langle\nabla_{u} u, u\right\rangle_{s} & =\sum_{\alpha=s}\left\langle D^{\alpha}\left(\nabla_{u} u\right), D^{\alpha} u\right\rangle \\
& =\sum_{\alpha=s} \sum_{\substack{|\beta| \leq s \leq s \\
\alpha-\beta \geq 0}}\left\langle c_{\alpha, \beta}\left(D^{\beta} \nabla u\right)\left(D^{\alpha-\beta} u\right), D^{\alpha} u\right\rangle \\
& =\sum_{\substack { \alpha=s \\
\begin{subarray}{c}{|\beta| \leq-s-1 \\
\alpha-\beta \geq 0{ \alpha = s \\
\begin{subarray} { c } { | \beta | \leq - s - 1 \\
\alpha - \beta \geq 0 } }\end{subarray}}\left\langle c_{\alpha, \beta}\left(D^{\beta} \nabla u\right)\left(D^{\alpha-\beta} u\right), D^{\alpha} u\right\rangle \\
& \leq C \sum_{m=0}^{s-1}\left|D^{m+1} u\right|_{L^{4}}\left|D^{s-m}\right|_{L^{4}}\left|D^{s} u\right| .
\end{aligned}
$$


where we set $|\beta|=m$ so that $|\alpha-\beta|=s-m$, and the last equality follows from the fact that $\left\langle\nabla_{u}\left(D^{s} u\right), D^{s} u\right\rangle=0$, since $\operatorname{div} u=0$. For $m=0, \ldots, s-1$, we use Eqs. (1.4) and (1.5) to estimate

$$
\begin{aligned}
& \left|D^{m+1} u\right|_{L^{4}\left|D^{s-m}\right|_{L^{4}}\left|D^{S} u\right|} \\
& \quad \leq C\left|D^{m+1} u\right|^{1 / 2}\left|D^{m+2} u\right|^{1 / 2}\left|D^{s-m} u\right|^{1 / 2}\left|D^{s-m+1} u\right|^{1 / 2}\left|D^{S} u\right| \\
& \quad \leq C|u|_{1}^{(s+1) / s}|u|_{s+1}^{2(s-1) / 2 s} .
\end{aligned}
$$

Using Young's inequality,

$$
a^{\lambda} b \leq \varepsilon a+\frac{C}{\varepsilon} b^{1 /(1-\lambda)}, \quad a, b>0, \quad 0<\lambda<1,
$$

it follows that

$$
\left\langle P \nabla_{u} u, u\right\rangle_{s} \leq \varepsilon|u|_{s+1}^{2}+\frac{C}{\varepsilon}|u|_{1}^{s+1} .
$$

For the next term, we have that

$$
\begin{aligned}
\left\langle\operatorname{Div}\left(\nabla d^{T} \cdot \nabla d\right), u\right\rangle_{s} & =\sum_{|\alpha|=s}\left\langle D^{\alpha} \operatorname{Div}\left(\nabla d^{T} \cdot \nabla d\right), D^{\alpha} u\right\rangle \\
& \leq C \sum_{\substack{|c|=s \\
|x|=s+1|\beta|<-s>0}} \int_{M}\left(D^{\beta} \nabla d\right)\left(D^{\gamma-\beta} \nabla d\right)\left(D^{\alpha} u\right) \mu \\
& \leq C \sum_{m=0}^{s+1}\left\{\left(D^{m+1} d\right)\left(D^{s-m+2} d\right),\left(D^{s} u\right)\right\rangle,
\end{aligned}
$$

where $m=|\beta|$. In the case that $m=1, \ldots, s$, Eq. (6.3) is bounded by

$$
\begin{aligned}
C \sum_{m=1}^{s}\left|D^{m+1} d\right|_{L^{4}}^{2}\left|D^{s-m+1} d\right|_{L^{4}}^{2} & \leq\left. C|d|_{2}\left|d_{s+2}\right| u\right|_{1} ^{1 / s}|u|_{s+1}^{(s-1) / s} \\
& \leq \varepsilon|d|_{s+2}^{2}+\frac{C}{\varepsilon}|d|_{2}^{2}|u|_{s}^{2 / s}|u|_{s+1}^{2(s-1) / s},
\end{aligned}
$$

where the first inequality follows from repeated use of Eq. (1.5), and the last inequality follows from $a b \leq \varepsilon a^{2}+(C / \varepsilon) b^{2}$, where $a, b>0$. One more application of Eq. (6.2) shows that Eq. (6.4) is bounded by

$$
\varepsilon|d|_{s+2}^{2}+\varepsilon|u|_{s+1}^{2}+\frac{C}{\varepsilon^{1+s}}|d|_{2}^{2 s}|u|_{1}^{2} .
$$


In the case that $m=0, s+1$, Eq. (6.3) is bounded by

$$
\begin{aligned}
C|d|_{s+2}|d|_{L^{4}}\left|D^{s} u\right|_{L^{4}} & \leq C|d|_{s+2}|d|_{2}|u|_{1}^{1 / 2 s}|u|_{s+1}^{(2 s-1) / 2 s} \\
& \leq \varepsilon|d|_{s+2}^{2}+\frac{C}{\varepsilon}|d|_{2}^{2}|u|_{1}^{1 / s}|u|_{s+1}^{2(s-1) / s} \\
& \leq \varepsilon|d|_{s+2}^{2}+\varepsilon|u|_{s+1}^{2}+\frac{C}{\varepsilon^{1+s}}|d|_{2}^{2 s}|u|_{1},
\end{aligned}
$$

where the first inequality follows from Eq. (1.5), and the last two inequalities follow from Eq. (6.2). It follows that

$$
\left\langle P \operatorname{Div}\left(\nabla d^{T} \cdot \nabla d\right), u\right\rangle_{s} \leq \varepsilon|u|_{s+1}^{2}+\varepsilon|d|_{s+2}^{2}+\frac{C}{\varepsilon^{1+s}}|d|_{2}^{2 s}\left(|u|_{1}+|u|_{1}^{2}\right) .
$$

We next compute that

$$
\begin{aligned}
\left\langle\nabla_{u} d, d\right\rangle_{s+1} & =\sum_{|\alpha|=s+1}\left\langle D^{\alpha}\left(\nabla_{u} d\right), D^{\alpha} u\right\rangle \\
& \leq C \sum_{|\alpha|=s+1} \sum_{\substack{|\beta| \leq s+1 \\
\alpha-\beta \geq 0}}\left\langle\left(D^{\beta} \nabla d\right)\left(D^{\alpha-\beta} u\right), D^{\alpha} \nabla d\right\rangle \\
& \leq C \sum_{m=0}^{s}\left\langle\left(D^{m+1} d\right)\left(D^{s-m+1} u\right), D^{s+1} d\right\rangle,
\end{aligned}
$$

since for $m=s+1$, we have that $\left\langle\nabla_{u}\left(D^{s+1} d\right), D^{s+1} d\right\rangle=0$. We estimate the case $m=0$ first in Eq. (6.5):

$$
\begin{aligned}
|D d|_{L^{4}}\left|D^{s+1} d\right|_{L^{4}}\left|D^{s+1} u\right| & \leq C|d|_{2}|u|_{s+1}|d|_{s+1}^{1 / 2}|d|_{s+2}^{1 / 2} \\
& \leq C|d|_{2}^{(2 s+1) / 2 s}|u|_{s+1}|d|_{s+2}^{(2 s-1) / 2 s} \\
& \leq \varepsilon|d|_{s+2}^{2}+\frac{C}{\varepsilon}\left(|d|_{2}^{(2 s+1) / 2}|u|_{s+1}\right)^{4 s /(2 s+1)} \\
& \leq \varepsilon|d|_{s+2}^{2}+\varepsilon|u|_{s+1}^{2}+\frac{C}{\varepsilon^{2 s+2}}|d|_{2}^{4 s+2}
\end{aligned}
$$

where the last two inequalities follow from two applications of the Young's inequality.

For the cases $1 \leq m \leq s$, Eq. (6.5) is bounded by $C\left|D^{m+1} d\right|_{L^{4}} \mid D^{s-m+1} \times$ $\left.u\right|_{L^{4}}|d|_{s+1}$, so by Eqs. (1.4) and (1.5), we find that for $m=1, \ldots, s$,

$$
\begin{aligned}
& \left|D^{m+1} d\right|^{1 / 2}\left|D^{m+2} d\right|^{1 / 2}\left|D^{s-m+1} u\right|^{1 / 2}\left|D^{s-m+2} u\right|^{1 / 2}\left|D^{s+1} d\right| \\
& \quad \leq C|d|_{2}^{(2 s-2 m+3) / 2 s}|d|_{s+2}^{(2 m+2 s-3) / 2 s}|u|_{1}^{(2 m-1) / 2 s}|u|_{s+1}^{(2 s-2 m+1) / 2 s} \\
& \quad \leq \varepsilon|d|_{s+2}^{2}+\left(\frac{C}{\varepsilon}|d|_{2}^{2}|u|_{1}^{(4 m-2) /(2 s-2 m+3)}\right)\left(|u|_{s+1}^{(4 s-4 m+2) /(2 s-2 m+3)}\right)
\end{aligned}
$$


where we have used Young's inequality for the last step. Another application of Young's inequality yields the estimate

$$
\begin{gathered}
\left\langle\nabla_{u} d, d\right\rangle_{s+1} \leq \varepsilon|d|_{s+2}^{2}+\varepsilon|u|_{s+1}^{2}+\frac{C}{\varepsilon^{(2 s-2 m+5) /(2 s-2 m+3)}}|d|_{2}^{4 /(2 s-2 m+3)}|u|_{1}^{2 m-1}, \\
m=1, \ldots, s .
\end{gathered}
$$

For the final nonlinear term, we have that

$$
\begin{aligned}
\langle f(d), d\rangle_{s+1} & \leq C \sum_{m=0}^{s+1} \sum_{n=0}^{m}\left\langle\left(D^{n} d\right)\left(D^{m-n} d\right)\left(D^{s+1-m} d\right), D^{s+1} d\right\rangle \\
& \leq\left|D^{n} d\right|_{L^{8}}\left|D^{m-n} d\right|_{L^{8}}\left|D^{s+1-m} d\right|_{L^{4} \mid} D^{s+1} d \mid .
\end{aligned}
$$

Using the estimate

$$
|v|_{L^{8}} \leq|v|_{L^{2}}^{5 / 8}|v|_{2}^{3 / 8}
$$

together with Eq. (1.5) and Young's inequality, we have that

$$
\langle f(d), d\rangle_{s+1} \leq C|d|_{2}^{2(s+2) / s}|d|_{s+2}^{2(s-2) / s} \leq \varepsilon|d|_{s+2}^{2}+\frac{C}{\varepsilon}|d|_{2}^{s+2} .
$$

\section{Letting}

$$
\begin{aligned}
\rho=C & {\left[\frac{1}{\varepsilon}\left(|d|_{2}^{s+2}+|u|_{1}^{s+1}\right)+\frac{1}{\varepsilon^{1+s}}|d|_{2}^{2 s}\left(|u|_{1}+|u|_{1}^{2}\right)+\frac{1}{\varepsilon^{2+2 s}}\left(|d|_{2}^{4 s+2}\right)\right.} \\
& \left.+\sum_{m=1}^{s} \frac{1}{\varepsilon^{(2 s-2 m+5) /(2 s-2 m+3)}}|d|_{2}^{4 /(2 s-2 m+3)}|u|_{1}^{2 m-1}\right],
\end{aligned}
$$

and taking $\varepsilon>0$ sufficiently small so that

$$
K=\min (\nu-4 \varepsilon, \gamma-4 \varepsilon)>0,
$$

the basic inequality Eq. (6.1) takes the form

$$
\frac{d}{d t}\left(|u|_{s}^{2}+|d|_{s+1}^{2}\right) \leq-K\left(|u|_{s+1}^{2}+|d|_{s+2}^{2}\right)+C \rho
$$

so that

$$
\frac{d}{d t}\left(|u|_{s}^{2}+|d|_{s+1}^{2}\right) \leq-\tilde{K}\left(|u|_{s}^{2}+|d|_{s+1}^{2}\right)+C \rho,
$$


for some constant $\tilde{K}>0$. Letting $c_{1}=C \tilde{K}>0$, the classical Gronwall lemma gives

$$
\left(|u|_{s}^{2}+|d|_{s+1}^{2}\right) \leq\left(\left|u_{0}\right|_{s}^{2}+\left|d_{0}\right|_{s+1}^{2}\right) e^{-c_{1} t}+C \rho\left(1-e^{-c_{1} t}\right),
$$

so that we have the uniform (in $t$ ) bound on $[0, T]$, and as an a priori estimate,

$$
\limsup _{t \rightarrow \infty}\left(|u|_{s}^{2}+|d|_{s+1}^{2}\right) \leq C \rho .
$$

Thus, since the time interval of existence from Theorem 4.1 only depends on the initial data, the a priori bound Eq. (6.9) together with the continuation property gives the global well-posedness result. Standard parabolic regularity results show that the solutions are smooth on the parabolic interior.

Integrating Eq. (6.7) from $t$ to $t+r$ and using Eq. (6.9), we have that

$$
K \int_{t}^{t+r}\left(|u(\tau, \cdot)|_{s+1}^{2}+|d(\tau, \cdot)|_{s+2}^{2}\right) d \tau \leq(1+r) C \rho .
$$

Replacing $s$ with $s+1$ in Eq. (6.8) and using Eq. (6.10) together with the uniform Gronwall Lemma 1.1, we obtain that $|u(t, \cdot)|_{s+1}^{2}+|d(t, \cdot)|_{s+2}^{2}$ is uniformly bounded for $t$ sufficiently large. This shows the existence of an absorbing set in $W^{s+1} \times H^{s+2}(T M)$; hence, we obtain using Theorem I.1.1 of Ref. [28], the global attractor that we asserted.

\subsection{Small Data Results}

Since we have shown that the liquid crystal model has the same structure as the Navier-Stokes equations, it is quite easy to establish the existence of unique global solutions in $H^{s}$ spaces, $s>n / 2+1$, for sufficiently small initial data.

Proposition 6.1. Let $\partial M=\emptyset$ and $s>n / 2+1$. For $u_{0} \in W^{s}, d_{0} \in H^{s+1}(M$, $\left.B^{\delta}\right)$ such that $\left|u_{0}\right|_{s}^{2}+\left|d_{0}\right|_{s+1}^{2}$ is sufficiently small, Eq. (1.1) has a global solution in

$$
\begin{aligned}
& u \in C^{0}\left([0, \infty), W^{s}\right) \cap C^{\infty}((0, \infty) \times M), \\
& d \in C^{0}\left([0, \infty), H^{s+1}\left(M, B^{\delta}\right)\right) \cap C^{\infty}((0, \infty) \times M) .
\end{aligned}
$$

Proof. Setting the parameters $v, \gamma, \lambda$ equal to one, using the basic Moser inequality

$$
|f \cdot g|_{s} \leq C\left[|f|_{L^{\infty}}|g|_{s}+|f|_{s}|g|_{L^{\infty}}\right]
$$


and the basic estimate Eq. (6.1), we find that for some positive constant $A, B, C$ and $\varepsilon$

$$
\begin{aligned}
\frac{d}{d t}\left[|u|_{s}^{2}+|d|_{s+1}^{2}\right] \leq & \left(-A+C|u|_{C^{1}}+\frac{C}{\varepsilon}|d|_{C^{1}}^{2}\right)|u|_{s}^{2} \\
& +\left(-B+2 \varepsilon+C\left(|u|_{C^{0}}+|d|_{C^{0}}^{2}\right)\right)|d|_{s+2}^{2} .
\end{aligned}
$$

We have used the fact that a Poincaré-type inequality exists on manifolds for which the Euler characteristic $\chi(M)$ does not vanish, which we assumed at the outset. This is not, however, a limitation, as we can always add the contribution of the $L^{2}$ norm and handle the estimate in a similar fashion (see, for example, Proposition 4.5 in Chapter 17 of Ref. [29]).

Taking $\epsilon$ small enough, and letting $K_{1}$ and $K_{2}$ denote a new pair of positive constants, we arrive at the inequality

$$
\frac{d}{d t}\left[|u|_{s}^{2}+|d|_{s+1}^{2}\right] \leq\left(-2 K_{1}+K_{2}\left(|u|_{C^{1}}+|d|_{C^{1}}^{2}\right)\right)\left(|u|_{s}^{2}+|d|_{s+1}^{2}\right) .
$$

Assume that $\left|u_{0}\right|_{s}^{2}+\left|d_{0}\right|_{s+1}^{2} \leq N$, and that $N>0$ is so small that

$$
|v|_{s}^{2}+|w|_{s+1}^{2} \leq N \Longrightarrow|v|_{C^{1}}+|w|_{C^{1}}^{2} \leq \frac{K_{1}}{K_{2}}
$$

As long as $|u(t, \cdot)|_{s}^{2}+|d(t, \cdot)|_{s+1}^{2} \leq N$, we have the inequality

$$
y^{\prime}(t) \leq-K_{1} y(t), \quad y(t)=|u(t, \cdot)|_{s}^{2}+|d(t, \cdot)|_{s+1}^{2},
$$

so that $y(t) \leq y(0) \leq N$. Thus, if we take $N$ small enough so that Eq. (6.11) holds, we have the global bound $y(t) \leq N$, and hence global existence.

\subsection{Manifolds with Boundary}

For a Riemannian manifold with boundary, the above (brute force) $H^{s}$ energy estimate does not work, because boundary terms arising from integration by parts on the diffusion term $v$ Div Def $u$ do not vanish. It is possible, however, to obtain estimates on $u_{t}$ and $d_{t}$ which provide the global well-posedness result.

We have that

$$
\begin{aligned}
u_{t t}= & v \text { Div Def } u_{t}-\nabla_{u_{t}} u-\nabla_{u} u_{t}-\operatorname{grad} p_{t}-\lambda \nabla d_{t}^{T} \cdot \hat{\Delta} d-\lambda \nabla d^{T} \cdot \hat{\Delta} d_{t} \\
& -\lambda g\left(R\left(e_{i}, \cdot\right) d_{t}, \nabla_{e_{i}} d\right)-\lambda g\left(R\left(e_{i}, \cdot\right) d, \nabla_{e_{i}} d_{t}\right)
\end{aligned}
$$


and

$$
d_{t t}=-\nabla_{u_{t}} d-\nabla_{u} d_{t}+\gamma \hat{\Delta} d_{t}-\gamma \operatorname{grad} f(d) \cdot d_{t} .
$$

Since $d_{t}=0$ on $\partial M$ we see that $(1 / 2)(d / d t)\left(\left|u_{t}\right|^{2}+\left|\nabla d_{t}\right|^{2}\right)=\left\langle u_{t}, u_{t t}\right\rangle-\left\langle\hat{\Delta} d_{t}\right.$, $\left.d_{t t}\right\rangle$. Standard interpolation combined with Young's inequality yields, for constants $c_{1}, c_{2}>0$,

$$
\frac{1}{2} \frac{d}{d t}\left(\left|u_{t}\right|^{2}+\left|\nabla d_{t}\right|^{2}\right)=c_{1}\left(|\operatorname{Def} u|^{2}+|\hat{\Delta} d|^{2}+c_{2}\right)\left(\left|u_{t}\right|^{2}+\left|\nabla d_{t}\right|^{2}\right) .
$$

We known that a smooth solution exists for $t \in(0, T]$, so on this time interval, Proposition 5.3 states that for almost all $t$ in this interval,

$$
u(t, \cdot) \in W^{1} \quad \text { and } d(t, \cdot) \in H^{2}(T M) .
$$

It follows that if $u_{0} \in W^{2}, d_{0} \in H^{3}(T M)$, and $h \in H^{5 / 2}(T \partial M)$, then $u_{t}(0) \in$ $L^{2}(T M)$ and $d_{t}(0) \in H_{0}^{1}(T M)$ so that

$$
u_{t} \in L^{\infty}\left([0, T], W^{0}\right) \quad \text { and } d_{t} \in L^{\infty}\left([0, T], H_{0}^{1}(T M)\right) .
$$

From Eq. (6.12), we claim that $\nabla_{u} d(t, \cdot)$ is in $H^{\delta}(T M)$ for almost all $t \in(0, T]$ and for $\delta \in(0,5 / 16)$. To see this, note that for $\varepsilon>0$

$$
w \mapsto w w: H^{p} \rightarrow H^{\theta(1+\varepsilon)}, \quad \text { where } p=\frac{1}{2}+\frac{1}{2} \theta(1+\varepsilon)+\varepsilon \theta .
$$

We set $\delta=\theta(1+\varepsilon)$, and, for example, set $\varepsilon=1 / 4$ and $\theta \leq 1 / 4$; then $\delta \in(0,5 / 16)$ and $p \geq 23 / 32$, so the claim is established. Using standard elliptic regularity on Eq. (5.3b), we see that $d \in H^{2+\delta}(T M)$, and the $H^{2+\delta}$. norm of $d$ only depends on the initial data and $M$.This shows that $\operatorname{Div}\left(\nabla d^{T} \cdot \nabla d\right)$ is in $L^{2}$ so that with Eq. (6.13), we see that $u$ is in $W^{2}$. By bootstrapping, we find that $d$ is in $H^{3}$, and the continuation argument shows that the unique solution may be continued for all time. If $h \in C^{\infty}(T \partial M)$, then both $u$ and $d$ are in $C^{\infty}((0, \infty) \times M)$. Thus, we have the following.

Theorem 6.2. Suppose that $u_{0} \in W^{2}$ and $d_{0} \in \dot{H}_{h}^{3}(T M)$. Then there exists a unique solution

$$
u \in C^{0}\left([0, \infty), W^{2}\right) \text { and } d \in C^{0}\left([0, \infty), \dot{H}_{h}^{3}(T M)\right) .
$$

If $h \in C^{\infty}(T \partial M)$, then both $u$ and $d$ are in $C^{\infty}((0, \infty) \times M)$.

For the weak solutions obtained in Ref. [16], Proposition 5.3 immediately proves the existence of a compact global attractor in $W^{0} \times$ $H_{h}^{1}(T M)$. A small data result may be obtained by a similar argument as in Proposition 6.1. 


\section{LAGRANGIAN AVERAGED LIQUID CRYSTALS}

As we described in the introduction, the director field $d$ describes locally the averaged direction of the constituent molecules; it is thus reasonable, and of practical and computational importance, to locally average the NavierStokes fluid motion as well. Recently, the Lagrangian averaged Navier-Stokes (LANS) equations were introduced as a model for the large scale Navier-Stokes fluid motion which averages or filters over the small, computationally unresolvable spatial scales (see Refs. [4,22] and the references therein). The LANS equations are parameterized by a small spatial scale $\alpha>0$-fluid motion at spatial scales smaller than $\alpha$ is averaged or filtered-out. There are two types of Lagrangian averaged Navier-Stokes equations: the isotropic and the anisotropic versions. We shall begin with the isotropic theory, and for simplicity of presentation, we shall assume that $M$ is flat.

The isotropic LANS equations for the mean velocity $u(t, x)$ are given by

$$
\begin{aligned}
& \partial_{t}\left(1-\alpha^{2} \Delta\right) u+\nabla_{u}\left(1-\alpha^{2} \Delta\right) u-\alpha^{2} \nabla u^{T} \cdot \Delta u \\
& \quad=-\operatorname{grad} p-v(1-\alpha 2 \Delta) A u \\
& \operatorname{div} u(t, x)=0, \\
& u=0 \quad \text { on } \partial M, \\
& u(0, x)=u_{0},
\end{aligned}
$$

where $A:=-P \Delta$ is the Stokes operator, and $P$ is the Leray projector. Eq. (7.1a) has an equivalent representation as

$$
\begin{aligned}
& \partial_{t} u+\nabla_{u} u+\mathcal{U}^{\alpha}(u)=-\left(1-\alpha^{2} \Delta\right)^{-1} \operatorname{grad} p-v A u \\
& \mathcal{U}^{\alpha}(u)=\alpha^{2}\left(1-\alpha^{2} \Delta\right)^{-1} \operatorname{Div}\left[\nabla u \cdot \nabla u^{T}+\nabla u \cdot \nabla u-\nabla u^{T} \cdot \nabla u\right] .
\end{aligned}
$$

When $\partial M=\emptyset$ the LANS equations take on a particularly familiar "subgrid-stress" form with Eq. (7.2) becoming

$$
\begin{aligned}
& \partial_{t} u+\nabla_{u} u+\operatorname{Div} \tau^{\alpha}(u)=-\operatorname{grad} p+v \Delta u \\
& \tau^{\alpha}(u)=\alpha^{2}\left(1-\alpha^{2} \Delta\right)^{-1}\left[\nabla u \cdot \nabla u^{T}+\nabla u \cdot \nabla u-\nabla u^{T} \cdot \nabla u\right],
\end{aligned}
$$

where $\tau^{\alpha}$ representing the sub-grid or "Reynolds stress."

The remarkable feature of the LANS equations is that, unlike the Reynolds averaged Navier-Stokes (RANS) equations or Large Eddy Simulation (LES) models of turbulence, no additional dissipation is put into the system. Infact, when $v=0$, the LANS equations conserve the 
Hamiltonian structure of the Euler equations with both a modified kinetic energy

$$
E^{\alpha}=\frac{1}{2} \int_{M}\left(|u|^{2}+2 \alpha^{2}|\operatorname{Def} u|^{2}\right) \mu
$$

and helicity

$$
H^{\alpha}=\int_{M} w \wedge d w, \quad w=\left(1-\alpha^{2} \Delta\right) u^{\mathrm{b}}, u^{\mathrm{b}}=g(u, \cdot),
$$

being conserved.

This is easiest to see from Eq. (7.3), where the only term that is added (to the NS equations) is $\operatorname{Div} \tau^{\alpha}(u)$; it is precisely this term which averages the small scales, and this is accomplished by the use of nonlinear dispersion as opposed to dissipation. A simple computation, which requires taking the $L^{2}$ inner-product of the LANS equations with $u$ when $v$ is set to zero, shows that Eq. (7.4) is conserved. Why is it so important not to over-dissipate the NS equations? The answer is two fold: first, the addition of artificial dissipation obviously and spuriously removes crucial small-scale features, and second, artificial viscosity, which is present in RANS or LES models, suppresses intermittency, a fundamental feature of fluid turbulence.

Mathematically, for all $\alpha>0$, the three-dimensional LANS equations are globally well-posed (see Refs. [11,23]), yet when the averaging parameter $\alpha$ is taken sufficiently small, computational simulations of LANS are statistically indistinguishable from the simulations of the NS equations. Furthermore, the LANS equations provide a tremendous computational savings as shown in simulations of both forced and decaying turbulence Refs. [3,24]. Finally, the LANS equations arise from a variational principle in the same fashion as the NS equations. We shall therefore base our development of the averaged liquid crystal equations on the LANS model, and introduce the following system of equations:

$$
\begin{aligned}
& u_{t}+v A u+\nabla_{u} u+\mathcal{U}^{\alpha}(u)=-\left(1-\alpha^{2} \Delta\right)^{-1}\left[\operatorname{grad} p+\operatorname{Div}\left(\nabla d^{T} \cdot \nabla d\right)\right] \\
& \operatorname{div} u(t, x)=0 \\
& d_{t}+\nabla_{u} d=\gamma\left(\Delta d-\frac{1}{\epsilon^{2}}\left(|d|^{2}-1\right) d\right) \\
& u=0 \text { on } \partial M, d=h \text { on } \partial M g(h, h)=1 \text { or } \partial M=\emptyset \\
& u(0, x)=u_{0}, d(0, x)=d_{0} \text { and }\left.d_{0}\right|_{\partial M}=h \text { if } \partial M \neq \emptyset
\end{aligned}
$$

where $\Delta$ denotes the componentwise Laplacian. 


\section{Averaged Variational Principle}

Following the notation of Section 2, we define the averaged action function $S^{\alpha}: \mathcal{D}_{\mu, D}^{s} \times H^{s+1}(T M) \cap H_{0}^{1}(T M) \rightarrow \mathbb{R}$ by

$$
\begin{aligned}
S^{\alpha}(\eta, d)= & \frac{1}{2} \int_{I} \int_{M}\{g(\eta(t, x))(u(t, \eta(t, x)), u(t, \eta(t, x))) \\
& +2 \alpha^{2} g(\eta(t, x))(\operatorname{Def} u(t, \eta(t, x)), \operatorname{Def} u(t, \eta(t, x))) \\
& +\lambda g(\eta(t, x))(\nabla[d(t, \eta(t, x))], \nabla[d(t, \eta(t, x))]) \\
& +2 F(d)\} \mu d t,
\end{aligned}
$$

where we suppress the explicit dependence of $u$ and $d$ on $\alpha$ and $\epsilon$.

Again, we see that Eq. (7.6a) arises as the first variation of the action function $S^{\alpha}$ with respect to $\eta$, and the remaining equations are identical to the original system Eq. (1.1). Note, however, that now $u$ is the mean velocity, and it is the mean flow $\eta$ which is transporting the director field $d$.

\section{Averaged Energy Law}

For simplicity, we shall present the formulation in the case that $\partial M=\emptyset$, although the more general case follows in the same fashion as we presented above. Following the notation of Section 2, we have the following basic averaged energy law:

$$
\begin{aligned}
& \frac{1 d}{2 d t}\left(|u|^{2}+\alpha^{2}|\nabla u|^{2}+\lambda|\nabla d|^{2}+2 \int_{M} F(d) \mu\right) \\
& \quad \leq-v\left(|\nabla u|^{2}+\alpha^{2}|\Delta u|^{2}\right)-\gamma \lambda|\Delta d-f(d)|^{2} .
\end{aligned}
$$

From Lemma 5.2 and (7.8), it follows that there exists $\bar{t}>0$, and some $\bar{\rho}_{0}>0$ which is independent of the initial data, such that

$$
|u(t, \cdot)|_{1}^{2}+|d|_{1}^{2} \leq \bar{\rho}_{0} \quad \forall t>\bar{t} .
$$

We see that the averaged energy law is, in some sense, more natural than the standard basic energy law Eq. (5.1) since the director field $d$ is no longer constrained to have one derivative greater regularity than the velocity of the fluid $u$ : both $u$ and $d$ now scale similarly.

Because of the a priori uniform bound of $u(t, \cdot)$ in $W^{1}$, it is very easy to obtain an a priori bound for $u(t, \cdot)$ in $W^{2}$ and $d \in H^{2}(T M)$ when the 
$\operatorname{dim}(M)=3$. We take the $L^{2}$ inner-product of $\left(1-\alpha^{2} \Delta\right)$ acting on (7.6a) with $\left(1-\alpha^{2} \Delta u\right)$; we then add to this the $L^{2}$ inner-product of $\Delta$ acting on Eq. (7.6a) with $\Delta d$, and use Lemma 5.2. Using similar estimates as the standard liquid crystal system, we obtain an a priori energy estimate, in fact an absorbing set, in $W^{2} \times H^{2}(T M)$, and by bootstrapping, we may easily obtain higher-order a priori estimates. Local well-posedness follows again from the contraction mapping argument that we gave in Theorem 4.1, so we have the following.

Theorem 7.1. For $n=2,3, s \geq 1, \partial M=\emptyset, \quad R=0$ and $u_{0} \in W^{s}, d_{0} \in$ $H^{s+1}(T M)$,

$$
u \in C^{0}\left([0, \infty), W^{s}\right), \quad d \in C^{0}\left([0, \infty), H^{s+1}(T M)\right.
$$

are solutions to the system of Eq. (1.1). Moreover, there exists a compact global attractor for the system Eq. (7.6) in $W^{s} \times H^{s+1}(T M)$.

We can generalize this theorem to Riemannian manifolds with boundary following the method in Ref. [23] and the previous section.

\section{CONCLUDING REMARKS}

\section{Gradient Flow Versus Damping}

We considered the $L^{2}$ gradient flow of the variation of the action function $S(\eta, d)$ with respect to $d$ in the director field Eq. (1.1e). In the liquid crystal literature, however, it is common to see a damped second-order equation for the director field (see Ref. [7] and references therein), which in the context of our simplified system would mean replacing $d_{t}$ with $\beta_{1} d_{t t}+\beta_{2} d_{t}$ for some constants $\beta_{1}$ and $\beta_{2}$. Of course, both types of equations have the identical stationary solutions, but in terms of stability, L. Simon's result ${ }^{[27]}$ guarantees that the damping term takes over. As far as parabolic estimates are concerned, it is easy to treat either type of equation, but we feel it is more natural to take the path of steepest descent in relaxing the orientation towards its preferred configuration.

\section{Lie Advection Versus Parallel Transport}

This remark concerns the coupling term $\nabla_{u} d$ in Eq. (1.1c). This term arises by considering the time derivative of $(d \circ \eta)(t, x):=d(t, \eta(t, x))$, where 
for each $t, \eta(t, \cdot)$ is a volume-preserving diffeomorphism in the topological group $\mathcal{D}_{\mu, D}^{s}$. In group-theoretic language, this suggests that the action of $\mathcal{D}_{\mu, D}^{s}$ on the vector space of director fields is on the right. The natural action of $\mathcal{D}_{\mu, D}^{s}$ on the vector space of director fields, however, is on the left, or by pushforward: instead of $d \circ \eta$, the natural action is $\eta_{*} d:=D \eta \cdot d \circ \eta^{-1}$. Taking the time derivative of $\left[\eta_{*} d\right](t, x)$ gives $\mathcal{L}_{u} d$, the Lie derivative of $d$ in the direction $u$. The Lie derivative $\mathcal{L}_{u} d=\nabla_{u} d-\nabla_{d} u$, and this is the actual term which is present in the Ericksen-Leslie model. A nontrivial extension of our analysis is required to analyze the system Eq. (1.1) with $\nabla_{u} d$ replaced by $\mathcal{L}_{u} d$, and this is accomplished in Ref. [6].

\section{Other Fluids Models}

Using our methodology, it is quite easy to study a number of other fluids models. For example, by replacing the Oseen-Frank energy with the Landau-Lifshitz free energy $1 / 2 \int_{M} A g(\nabla M, \nabla M) \mu$, where $M$ is the direction of magnetization in a cubic ferromagnet, we can obtain an almost identical system of PDEs. Similarly, if we replace the vector $d$ in our action function $S$ with a scalar field $\phi$, and replace the $L^{2}$ gradient flow in Eq. (1.1c) with $H^{-1}$ gradient flow, we obtain a model of two-phase flow whose interface moves via motion by mean curvature (see Ref. [20]). This model consists of a coupled Navier-Stokes Cahn-Hilliard system, where the interface is governed by surface tension.

\section{The Defect Law in the Limit as $\epsilon \rightarrow 0$}

We considered the GL penalization of the Oseen-Frank energy law so as to obtain finite-energy minimizers, but we have yet to consider the limit of our solutions as $\epsilon \rightarrow 0$. It remains an open problem to characterize the dynamical law of the GL vortices when coupled to the Navier-Stokes motion. Following the pioneering work in Ref. $[2,18]$ on the dynamical law of the GL vortices, the location of the $j$ th vortex, $a_{j}$, solves the distributional equation

$$
\partial_{t} a_{j}+\operatorname{div}\left(a_{j} u\right)=\frac{\delta W}{\delta a_{j}},
$$

where $u$ simultaneously solves the Navier-Stokes equations, and $W=\sum_{i \neq j}$ $\log \left|x_{i}-x_{j}\right|$ is the renormalized energy. A rigorous defect law exists when the velocity $u$ comes from the LANS equations, because in that case, $u$ is 
uniformly in $H^{1}$ with respect to the penalization parameter $\epsilon>0$ (see Ref. [15]).

\section{ACKNOWLEDGMENTS}

I am grateful to Chun Liu for introducing me to the beautiful theory of liquid crystals, and to Daniel Coutand for carefully reading the manuscript. I would also like to thank John Ball, Dick James, and Stefan Müller for inviting me to the Oberwolfach conference Mathematical Continuum Mechanics where much of this work was initiated. Research was partially supported by the NSF-KDI grant ATM-98-73133 and the Alfred P. Sloan Foundation Research Fellowship.

\section{REFERENCES}

1. Agmon, S.; Douglis, A.; Nirenberg, L. Estimates Near the Boundary for Solutions of Elliptic Partial Differential Equations Satisfying General Boundary Conditions II. Comm. Pure Appl. Math. 1964, 17, 35-72.

2. Bethuel, F.; Brezis, H.; Hélein, F. Ginzburg-Landau Vortices, Birkhäuser, 1994.

3. Chen, S.Y.; Foias, C.; Holm, D.D.; Olson, E.J.; Titi, E.S.; Wynne, S. The Camassa-Holm Equations and Turbulence. Physica D 1999, 133, 49-65.

4. Chen, S.Y.; Foias, C.; Holm, D.D.; Olson, E.J.; Titi, E.S.; Wynne, S. The Camassa-Holm Equations and Turbulence in Pipes and Channels. Phys. Fluids 1999, 11, 2343-2353.

5. Chen, M.Y.; Struwe, M. Existence and Partial Regularity Results for the Heat Flow for Harmonic Maps. Math. Z., 1989, 201, 83-103.

6. Coutand, D.; Shkoller, S. Well-Posedness of the Full Ericksen-Leslie Model of Nematic Liquid Crystals. C.R. Acad. Sci. Paris Ser. I Math. 2001, 333, 919-924.

7. De Gennes, P.G.; Prost, J. The Physics of Liquid Crystals, 2nd Ed.; Clarendon Press: Oxford, 1993.

8. Ebin, D.; Marsden, J. Groups of Diffeomorphisms and the Motion of an Incompressible Fluid. Ann. Math. 1970, 92, 102-163.

9. Ericksen, J. Conservation Laws for Liquid Crystals. Trans. Soc. Rheol. 1961, 5, 22-34.

10. Ericksen, J. Equilibrium Theory for Liquid Crystals. In: Advances in Liquid Crystals, Vol. 2; Brown, G. Ed.; Academic Press: New York, 1975; pp. 233-398. 
11. Foias, C.; Holm, D.D.; Titi, E.S. The Three Dimensional Viscous Cammassa-Holm Equations and Their Relation to the NavierStokes Equations and Turbulence Theory. J. Dyn. Diff. Eq. 2002, 1, $1-35$.

12. Gliklikh, Y. Global Analysis in Mathematical Physics. Springer-Verlag, 1997.

13. Leslie, F.M. Theory of Flow Phenomena in Liquid Crystals. In Advances in Liquid Crystals, Vol. 4; Brown, G.; Ed.; Academic Press: New York, 1979; pp. 1-81.

14. Lin, F.-H. Nonlinear Theory of Defects in Nematic Liquid Crystals: Phase Transition and Flow Phenomena. Comm. Pure Appl. Math. 1989, 42, 789-814.

15. Lin, F.-H., Unpublished manuscript.

16. Lin, F.-H., Liu, C. Nonparabolic Dissipative Systems Modeling the Flow of Liquid Crystals. Comm. Pure Appl. Math. 1995, 48, 501-537.

17. Lin, F.-H., Liu, C. Existence of Solutions for the Ericksen-Leslie System. Arch. Ration. Mech. Anal. 2000, 154, 135-156.

18. Lin, F.-H.; Xin, J.X. On the Dynamical Law of the Ginzburg-Landau Vortices on the Plane. Comm. Pure Appl. Math. 1999, 52, 1189-1212.

19. Liu, C. Private Communication.

20. Liu, C.; Shkoller, S. Global Existence and the Sharp Interface Limit of the Phase-field Navier-Stokes Equations, in preparation.

21. Liu, C.; Walkington, N.J. Approximation of Liquid Crystal Flows. SIAM J. Numer. Anal. 2000, 37, 725-741.

22. Marsden, J.E.; Shkoller, S. The Anisotropic Lagrangian Averaged Euler and Navier-Stokes Equations. Arch. Rat. Mech. Anal. 2001, to appear.

23. Marsden, J.E.; Shkoller, S. Global Well-Posedness for the Lagrangian Averaged Navier-Stokes (LANS- $\alpha$ ) Equations on Bounded Domains. Proc. Roy. Soc. Philos. Trans. Ser. A Math Phys. Eng. Sci.: London, 2001, 359 (1784), 1149-1468.

24. Mohseni, K.; Shkoller, S.; Kosović, B.; Marsden, J.E.; Carati, D.; Wray, A.; Rogallo, R. Numerical Simulations of Homogeneous Turbulence Using the Lagrangian Averaged Navier-Stokes Equations, Center for Turbulence Research. Proceedings of the Summer Program 2000, 271-283.

25. Nirenberg, L. On Elliptic Partial Differential Equations. Ann. Scuola. Norm. Sup. Pisa 1959, 13, 115-162.

26. Shkoller, S. Analysis on Groups of Diffeomorphisms of Manifolds with Boundary and the Averaged Motion of a Fluid. J. Differential Geom. 2000, 55 (1), 145-191. 
27. Simon, L. Asymptotics for a Class of Nonlinear Evolution Equations with Applications to Geometric Problems. Ann. Math. 1983, 118, 525-572.

28. Temam, R. Infinite-Dimensional Dynamic Systems in Mechanics and Physics, Applied Mathematical Sciences, Vol. 68, Springer-Verlag: New York, 1988.

29. Taylor, M.E. Partial Differential Equations, III. Nonlinear Equations. Corrected reprint of the 1996 original. Applied Mathematical Sciences, Vol. 117, Springer-Verlag: New York, 1997.

Received February 2001

Revised April 2001 
